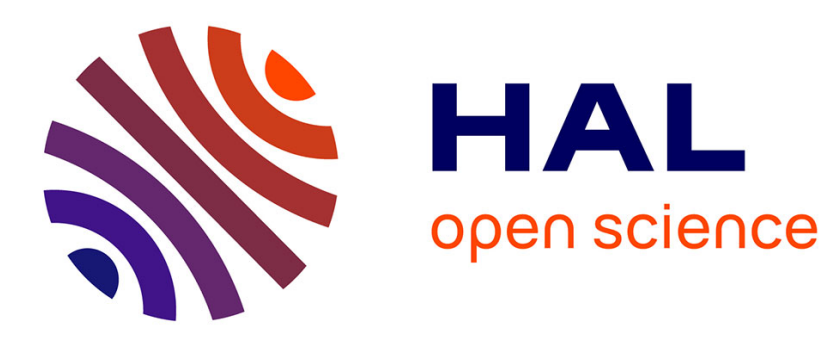

\title{
Vortex Dynamics in the Wake of Three Generic Types of Free-Stream Turbines
}

Matthieu Boudreau, Guy Dumas

\section{To cite this version:}

Matthieu Boudreau, Guy Dumas. Vortex Dynamics in the Wake of Three Generic Types of FreeStream Turbines. 16th International Symposium on Transport Phenomena and Dynamics of Rotating Machinery, Apr 2016, Honolulu, United States. hal-01891319

\section{HAL Id: hal-01891319 https://hal.science/hal-01891319}

Submitted on 9 Oct 2018

HAL is a multi-disciplinary open access archive for the deposit and dissemination of scientific research documents, whether they are published or not. The documents may come from teaching and research institutions in France or abroad, or from public or private research centers.
L'archive ouverte pluridisciplinaire HAL, est destinée au dépôt et à la diffusion de documents scientifiques de niveau recherche, publiés ou non, émanant des établissements d'enseignement et de recherche français ou étrangers, des laboratoires publics ou privés. 


\title{
Vortex Dynamics in the Wake of Three Generic Types of Free-Stream Turbines
}

\author{
Matthieu Boudreau ${ }^{1}$, Guy Dumas ${ }^{1 *}$
}

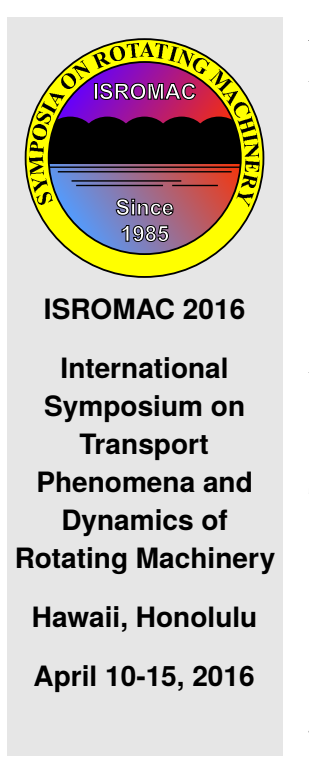

\begin{abstract}
An analysis of the vortex dynamics in the wake of three different free-stream turbine concepts is conducted to gain a better understanding of the main processes affecting the energy recovery in their wakes. The turbine technologies considered are the axial-flow turbine (AFT), the cross-flow turbine (CFT), also known as the H-Darrieus turbine, and the oscillating-foil turbine (OFT). The analysis is performed on single turbines facing a uniform oncoming flow and operating near their optimal efficiency conditions at a Reynolds number of $10^{7}$. Three-dimensional Delayed Detached-Eddy Simulations (DDES) are carried out using a commercial finite-volume Navier-Stokes solver. It is found that the wake dynamics of the AFT is significantly affected by the triggering of an instability while that of the CFT and the OFT are mainly governed by the mean flow field stemming from the tip vortices' induction.

\section{Keywords}

Turbine - Wake - Vortex dynamics - Axial-flow — Cross-flow — Oscillating-foil
\end{abstract}

\section{INTRODUCTION}

Several innovative renewable energy sources are being developed nowadays. Among them, the wind energy and the marine current sectors are receiving a lot of attention because of their great potential. Various turbine designs have been proposed so far, including the well-known axial-flow turbine and the cross-flow turbine, as well as the oscillating-foil turbine.

Despite the fact that a tremendous amount of work has already been devoted to the development of these technologies, the vortex dynamics in turbines' wakes at high Reynolds numbers is very complex and is not fully understood yet. Developing a better knowledge about this dynamics is crucial as it allows to gain better insights into the physics at play, especially regarding the wake recovery. Cutting-edge Delayed Detached-Eddy Simulations (DDES) of the three turbine technologies mentioned above are carried out in this work to achieve this task.

The axial-flow turbine's wake has been analyzed by Sherry et al. [1] who performed PIV measurements in the wake of a turbine facing a uniform oncoming flow. They focused on the vortex instabilities occurring in the wake, which was found to be strongly dependent on the tip speed ratio. Chamorro et al. [2] also observed a similar instability in the wake of an AFT located in a turbulent boundary layer flow mimicking a marine boundary layer. The stereoscopic particle image velocimetry (SPIV) measurements of Lignarolo et al. [3] have suggested that the enhanced mixing caused by the tip vortices' instability has a pronounced effect on the momentum recovery. However, their measurements were limited to the near wake, i.e., up to only 5 diameters downstream of the turbine.

Regarding the cross-flow turbines, several studies focused on the evolution of the shed vorticity and the tip vortices in their wake and highlighted the fact that the tip vortices ejected from the CFT blades tend to convect toward the wake center in the spanwise direction $[4,5,6,7,8,9]$. The same observation was also made more recently by Tescione et al. [10] who used the PIV technique to observe in details the flow field in the near wake of an H-Darrieus CFT turbine. However, their measurements only covered the first three diameters downstream of the turbine.

Deng et al. [11] showed qualitatively that the wake topology of an oscillating cross-flow turbine operating at a Reynolds number of 1,100 is strongly related to the blade's aspect ratio. Moreover, it is worth mentioning that the wakes of a flapping foil $[12,13]$ and that of a pitching panel $[14,15]$ used in the propulsion regime at low Reynolds numbers $\left(\operatorname{Re} \sim 10^{2}-10^{3}\right)$ have both been found to widen in the transverse direction but to contract in the spanwise direction, hence corroborating the fact that the three-dimensional effects significantly affect the wake dynamics of an oscillating foil. However, no studies of the three-dimensional oscillating cross-flow turbine's wake have been conducted at high Reynolds numbers so far.

The objective of the current work is to bring more light on the vortex dynamics observed in turbines wakes. A particular emphasis is placed on how this dynamics affects the wake recovery. The numerical methodology is presented in $\S 1$ and the results are shown and discussed in $\S 2$. 


\section{METHODS}

\subsection{Geometric characteristics and operating pa- rameters}

The axial-flow turbine (AFT) $[16,17]$ is characterized by blades rotating at constant angular speed about an axis aligned with the direction of the flow. The cross-flow turbine (CFT) concept [18] also involves rotating blades except that they rotate around an axis perpendicular to the flow direction. The axis may be either horizontal or vertical, which explains why the name vertical-axis turbine is also commonly used to refer to cross-flow turbines. The blades of the oscillating-foil turbine (OFT) undergo both pitching and heaving motions in a plane perpendicular to the oncoming flow. These motions are constrained to be sinusoidal and the phase lag between both motions is enforced through a mechanical linkage making the turbine a one degree-of-freedom device [19]. Outlines of the three concepts are shown in Fig. 1. Note that the turbine's characteristic length, $D$, is illustrated for each turbine concept. It corresponds to the turbine's diameter in the case of the AFT and the CFT and to the overall extent of the blade motion in the case of the OFT.

In this study, the operating conditions of the three turbine concepts have been chosen so that the turbines operate near their optimal efficiency conditions. The axial-flow turbine operates at a tip speed ratio $(\lambda)$ of 3.5 , defined as:

$$
\lambda=\frac{\omega D}{2 U_{\infty}},
$$

where $\omega$ is the turbine's angular velocity and $U_{\infty}$ is the freestream velocity. The geometry of the AFT's blades, based on a thicker version of the SD8020 profile, has been developed by the University of Victoria [20] in the context of a study for the Marine Energy Standards (TC114) [21]. The crossflow turbine considered in this work is an H-Darrieus turbine consisting of simple straight blades. The rotating shaft and connecting arms are not included in this study. More precisely, the CFT investigated corresponds to one of the single-bladed turbines studied by Gosselin et al. [22], which is characterized by a NACA0015 profile, a blade's aspect ratio $(b / c)$ of 15 , a diameter to chord length ratio of 7 and a tip speed ratio of 4.25 . As shown in Fig. 1, the blade's attach point $x_{p}$ is at the third of the chord length. The OFT considered in this work has been chosen to correspond to the one analyzed by Kinsey \& Dumas [23] with a blade's aspect ratio of 5, an overall extent of the blade motion to chord ratio $D / c$ of 2.55 and operating at a reduced frequency $\left(f^{*}\right)$ of 0.14 , as defined by:

$$
f^{*}=\frac{f c}{U_{\infty}},
$$

where $c$ is the chord length and $f$ is the oscillation frequency of both sinusoidal motions, namely the pitching and the heaving motions. The distance between the leading edge of the blade and its attach point $\left(x_{p}\right)$ also corresponds to a third of the chord length.

For comparison purposes, all three simulations have been conducted at a Reynolds number $\operatorname{Re}_{D}$ of $10^{7}$ based on the
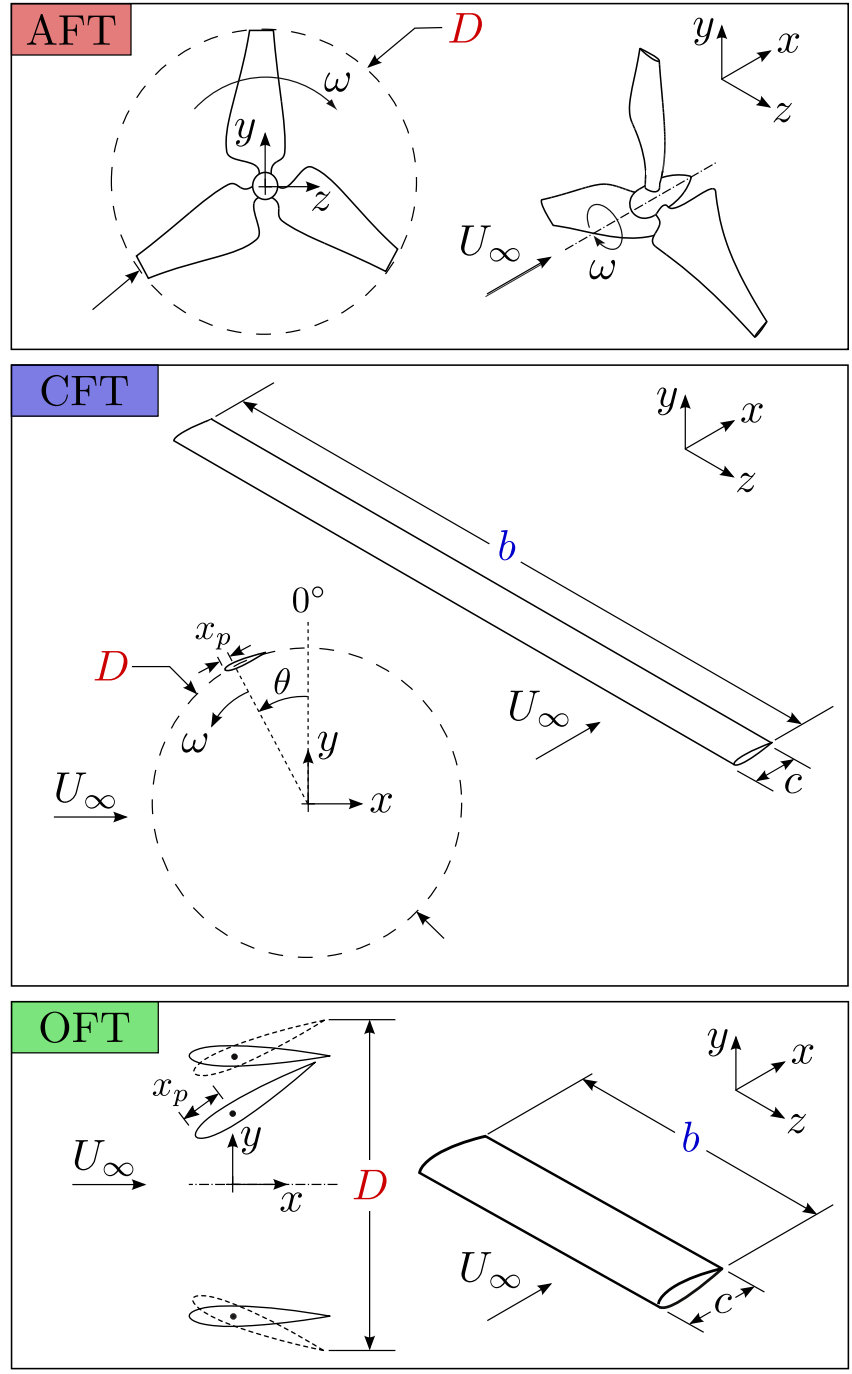

Figure 1. Outline and main parameters of the axial-flow turbine (top), the cross-flow turbine (middle) and the oscillatingfoil turbine (bottom) considered in this study.

turbine's characteristic length and the freestream velocity:

$$
\operatorname{Re}_{D}=\frac{U_{\infty} D}{v} .
$$

This value roughly corresponds to a middle-size turbine. However, at such a high Reynolds number, the results presented in this study are expected to be essentially independent of an increase in the Reynolds number [24, 25]. The present observations and conclusions should therefore also apply to large-scale turbines.

Note that the blade's aspect ratio of the CFT is higher than that of the OFT but the turbine's aspect ratio $(b / D)$ of these two turbine concepts is very similar $(b / D \approx 2$, see Fig. 1$)$.

\subsection{Turbulence modeling}

To circumvent the limitations of the RANS approach for wake flow simulation [26] and the prohibitive computational cost of a full LES simulation, we use in this study the Delayed 
Detached-Eddy simulation (DDES) approach [27, 28]. This hybrid methodology combines an efficient RANS approach in the attached flow regions near the walls and a more complete LES approach in the free regions of the flow away from the walls. The original DDES formulation is based on the one-equation Spalart-Allmaras turbulence model [29]. The only turbulent transport equation of this model, involving a modified viscosity scale $(\tilde{v})$, is adapted to switch from a RANS formulation to a LES formulation. One replaces the distance $d$ between a point in the domain and the nearest solid surface by the parameter $\tilde{d}$ defined as:

$$
\tilde{d}=d-f_{d} \max \left(0, d-C_{D E S} \cdot \Delta\right),
$$

where $f_{d}$ is a filter function designed to take a value of 0 in attached boundary layers (RANS region) and a value of 1 in zones where the flow is separated (LES region), $C_{D E S}$ is a constant equal to 0.65 and $\Delta$ is a length scale related to the local grid spacing $[28,30]$ :

$$
\Delta=\max (\Delta x, \Delta y, \Delta z) .
$$

The value of the eddy viscosity $\left(v_{t}\right)$ is then obtained from the modified viscosity ( $\tilde{v})$ through an empirical relation [29]. As a complete discussion on DDES modeling is out of the scope of this paper, the reader is referred to previous works that consider this matter more extensively [27, 28, 31].

\subsection{Numerics}

The finite-volume Navier-Stokes solver included in the CDAdapco $^{\text {TM }}$ STAR-CCM+ ${ }^{\circledR}$ software has been used to carry out the simulations [32]. Second-order schemes have been used for both the diffusive and the convective fluxes as well as for the temporal discretization. The scheme used for the convective flux is a hybrid bounded central-differencing scheme which combines upwind and centered schemes based on the procedure proposed by Travin et al. [33] for DDES simulations. For the pressure-velocity coupling, a segregated approach using the SIMPLE algorithm has been adopted.

The boundaries of the computational domain, shown in Fig. 2, are located far enough from the turbine so that it can be considered unconfined with very small blockage ratios [34]:

$$
\epsilon=A_{\text {turbine }} / A_{\text {domain }} \lesssim 0.003
$$

At the inlet boundary ( $15 D$ upstream of the turbine), we impose a uniform and constant upstream velocity and a specified turbulent viscosity ratio of three $\left(v_{t} / v=3\right)$. The corresponding upstream value of the modified viscosity ratio $(\tilde{v} / v)$ is 6.65 , which agrees with the recommendation of Spalart \& Rumsey [35] made to ensure that the model is in fully turbulent mode. A uniform zero static pressure is imposed at the outlet far downstream ( $30 \mathrm{D}$ from the turbine). Symmetry boundary conditions have been used on the four remaining surfaces of the computational domain. For the initial condition, we use uniform fields throughout the domain for velocity, pressure and turbulent viscosity ratio with values corresponding to the inlet and outlet boundary conditions.

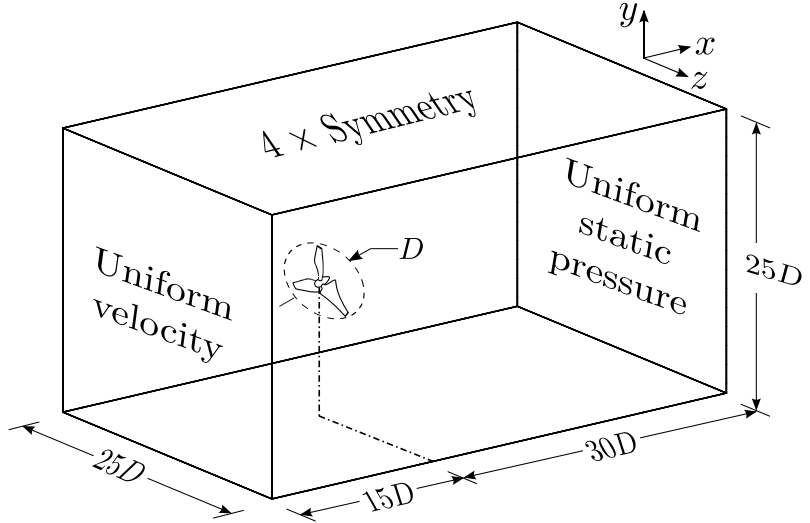

Figure 2. Computational domain and boundary conditions. The turbine's center is located in the center of the domain in the $y$ and $z$ directions. The axial-flow turbine shown is this figure has been enlarged tenfold for the sake of clarity.

A chimera grid technique has been used to deal with the blades' movement specific to each turbine concept [32]. In each case, the background mesh is composed of several mesh regions each consisting of orthogonal cubic cells having a specified uniform size. The resolution is kept high with small cell sizes in the whole wake region over $20 D$ downstream of the turbine. This refined region extends over $20 D$ even though the detailed analysis of the wake dynamics is restricted to the first $12 D$ in order to minimize the effects of the downstream mesh coarsening. For the moving mesh region of the AFT concept, an automatic polyhedral mesh technique with prism layers near the turbine surfaces have been used. For the moving mesh regions of the CFT and the OFT concepts, a two-dimensional mesh consisting of quad elements has been extruded in the spanwise $z$ direction (e.g., see Fig. 3). For all three cases, the near-wall resolution is fine enough to ensure that the maximum dimensionless normal wall distance $\left(y^{+}\right)$remains around unity throughout the whole turbine's cycle. The resulting total numbers of cells used for each of the three turbine concepts are given in Table 1 along with an approximation of the CPU resources required to perform the computations.

To ensure that the local Courant number is under one all along the turbine cycles and everywhere, including in the moving mesh regions where we get the highest values, the time step $(\Delta t)$ is set such that the turbine's cycle is divided into

Table 1. Total number of cells for the mesh of each turbine concept as well as the typical CPU resources required.

\begin{tabular}{lrrr}
\hline Case & Number of cells & Nb of cores & Runtime/cycle \\
\hline AFT & $62,118,147$ & 156 & $53 \mathrm{~h}$ \\
CFT & $133,761,744$ & 336 & $37 \mathrm{~h}$ \\
OFT & $19,367,422$ & 128 & $27 \mathrm{~h}$ \\
\hline
\end{tabular}




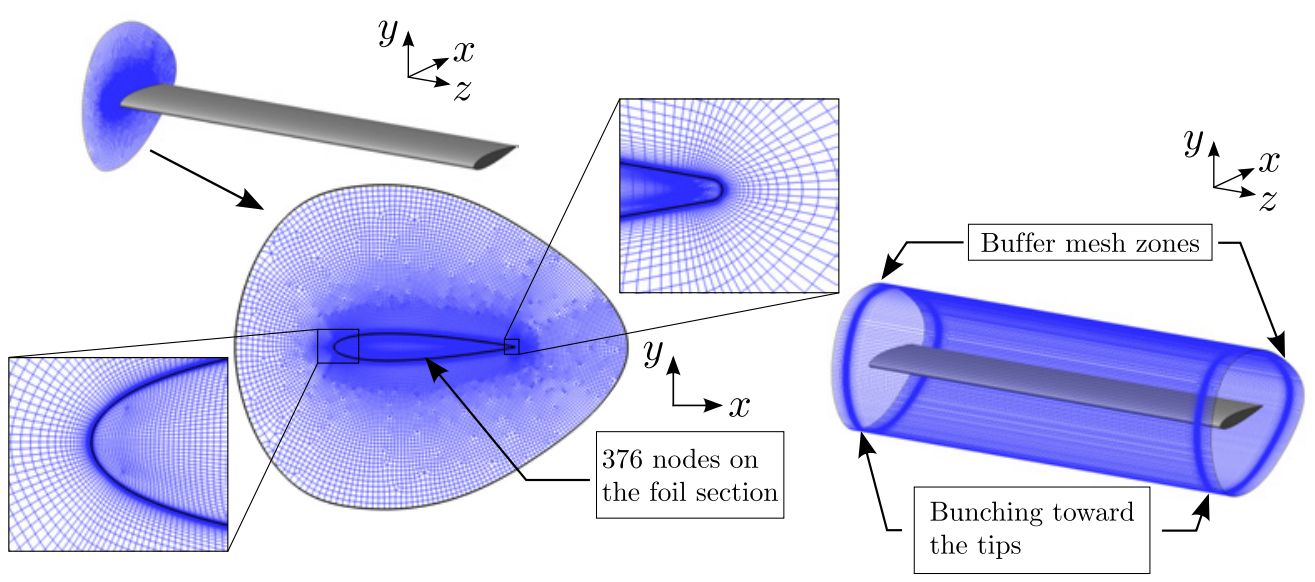

Figure 3. Moving mesh region of the OFT case.

1000 time steps. This is consistent with the recommendations made by Spalart [36] and by Mockett et al. [37] for good accuracy in regions of the flow resolved in LES mode in the context of DDES simulations. Assuming that the velocity in the wake roughly corresponds to $U_{\infty} / 2$, the local Courant number in the most refined region of the background grid is around 0.1 for all turbine technologies.

The spatial and temporal resolutions of the simulations in this study have already been proven to be adequate in previous investigations on turbines [22, 23, 38]. Nonetheless, two more simulations for each turbine concept have been carried out in order to confirm the resolution adequacy. One simulation has been performed using a mesh two times coarser than the base case in all three direction and another simulation has been performed with the mesh's base case and a temporal resolution $50 \%$ finer. For all three turbine concepts, the differences observed between the three simulations were found to be small enough for the base case simulation to be considered independent of the spatial and temporal discretizations used. The resolution sensitivity study of the mean streamwise velocity is shown in Table 2 as an example. To perform this study, the mean streamwise velocity has been measured on the wake centerline between $D$ and $12 D$ downstream of the turbines' center. The maximum local difference with respect to the base case and the absolute difference averaged along the entire line are given as a fraction of $U_{\infty}$.

Before starting recording any data for the wake statistical analysis over a certain amount of cycles $\left(\mathrm{N}_{\text {recorded }}\right)$, an initial transitory phase must be simulated. One can estimate this duration, given in a number of cycles $\left(\mathrm{N}_{\text {start }}\right)$ in Table 3 , if one assumes that the characteristic velocity in the wake is roughly $U_{\infty} / 2$ and that one needs to convect the initial flow conditions over at least $12 D$. In this study, we let 6 more cycles elapsed beyond that time to account for the fact that the few first cycles are not representative of the final statistically-converged cycles. In each case, good statistical convergence has been verified.
Table 2. Resolution sensitivity study of the mean streamwise velocity on the wake centerline with a mesh two times coarser and a time step $50 \%$ smaller.

\begin{tabular}{llcc}
\hline Case & & Max difference & Mean difference \\
\hline \multirow{2}{*}{ AFT } & Coarse mesh & $-5.6 \%$ & $1.9 \%$ \\
& Fine time step & $-6.5 \%$ & $2.5 \%$ \\
\hline \multirow{2}{*}{ CFT } & Coarse mesh & $-8.1 \%$ & $3.2 \%$ \\
& Fine time step & $-3.2 \%$ & $1.3 \%$ \\
\hline \multirow{2}{*}{ OFT } & Coarse mesh & $-5.0 \%$ & $2.5 \%$ \\
& Fine time step & $-6.2 \%$ & $2.9 \%$ \\
\hline
\end{tabular}

Table 3. Cycles recorded for statistical analysis.

\begin{tabular}{lrr}
\hline & $\mathrm{N}_{\text {start }}$ & $\mathrm{N}_{\text {recorded }}$ \\
\hline AFT & 36 & 30 \\
CFT & 39 & 22 \\
OFT & 16 & 45 \\
\hline
\end{tabular}

\section{RESULTS AND DISCUSSION}

\subsection{Axial-flow turbine}

The vortex dynamics in the wake of the AFT may be infered from the visualization of Fig. 4. One observes in the near wake the presence of a helicoidal vortex system formed by the root vortices in the center, and a system formed by the tip vortices at the edge of the wake. The well organized structures in the near wake become unstable at about $5 D$ downstream of the turbine's center and the whole vortex system is seen to breakdown rapidely thereafter. This behavior has been reported in the past by several contributors [1, 2, 3, 39].

The wake velocity deficit recovery of the axial-flow turbine is made visible on Fig. 5a which shows the mean streamwise 


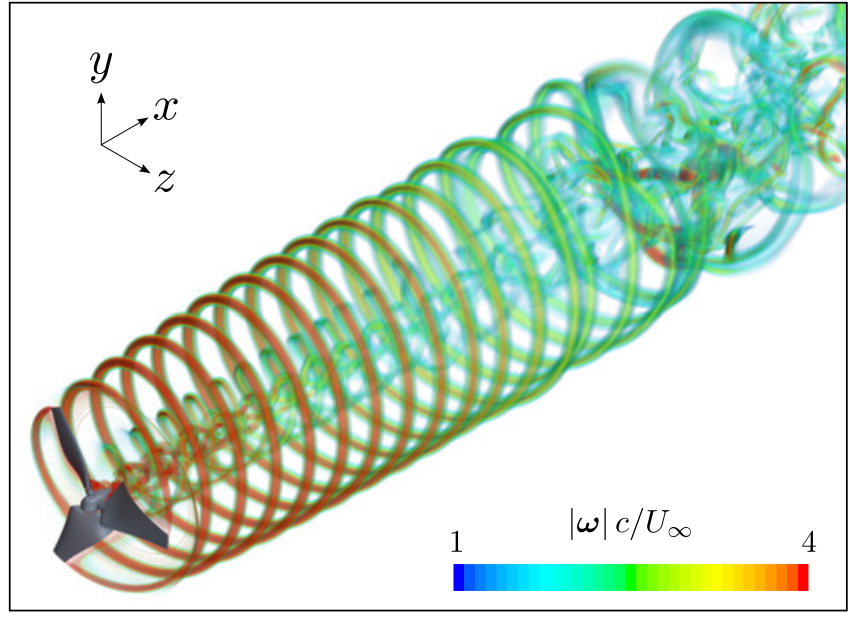

Figure 4. Volume rendering of the vorticity magnitude in the wake of the axial-flow turbine.

velocity contours in a plane passing through the turbine's axis of rotation at $z / D=0$. First, one observes that the maximum velocity deficit is found at approximately $3.5 \mathrm{D}$ downstream of the turbine's center and not immediately behind the turbine. One further notices that the mean wake is axisymmetric and characterized by a high velocity region in the center surrounded by an annular-shaped velocity deficit region. This structure has also been observed in several previous studies [1, $39,40,41,42]$. The presence of strong root vortices in the near wake explains the annular-shaped velocity deficit region observed in Fig. 5a. Indeed, those vortices are responsible for inducing a positive streamwise velocity in the wake's central core. Further downstream, at approximately $6 D$, one notices a rapid velocity recovery which is due to the enhanced turbulent mixing resulting from the breakdown of the root and tip vortex systems mentioned earlier. This yields the observed transition from an annular-shaped velocity deficit to a more usual Gaussian-shaped velocity deficit.

It thus seems that the AFT's wake recovery is dependent on the development of a flow instability which is responsible for inducing significant turbulent transport. This suggests that this recovery processs is highly sensitive to the perturbations in the upstream flow and to its turbulence intensity, as already pointed out in previous studies [ $43,44,45,46,47,48]$. It is nonetheless expected that the main mechanism affecting this wake recovery, namely the vortex system breakdown, stays the dominant process even with a more turbulent oncoming flow. The precise location where the breakdown occurs however should be affected [40, 48, 49]. Indeed, the higher the turbulence level, the sooner the instability may be triggered and the recovery occuring. This most likely explains why higher recovery rates have been reported for the wake of a turbine located downstream in the wake of another turbine $[40,50]$.

It is also very important to notice here that the helicoidal vortex system in the near wake of the AFT does not induce significant radial velocity on average. This is shown in Fig. 5b for the present turbine but this fact was also noted in other

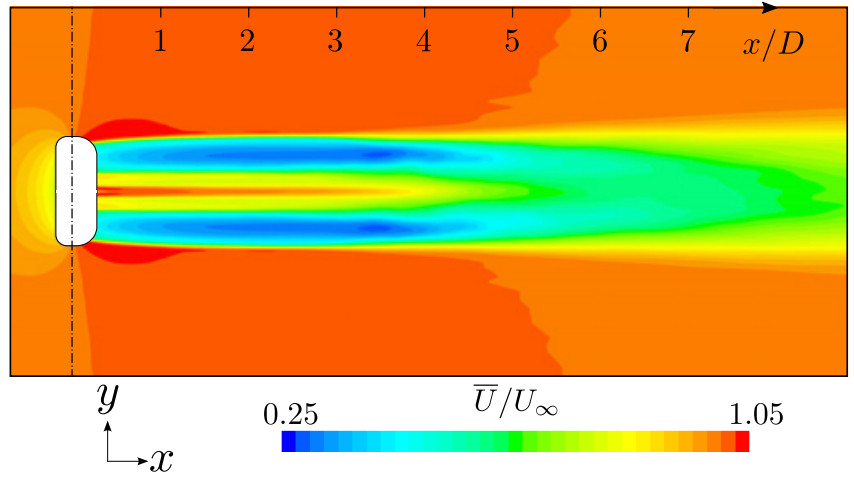

(a) Mean streamwise (axial) velocity component.

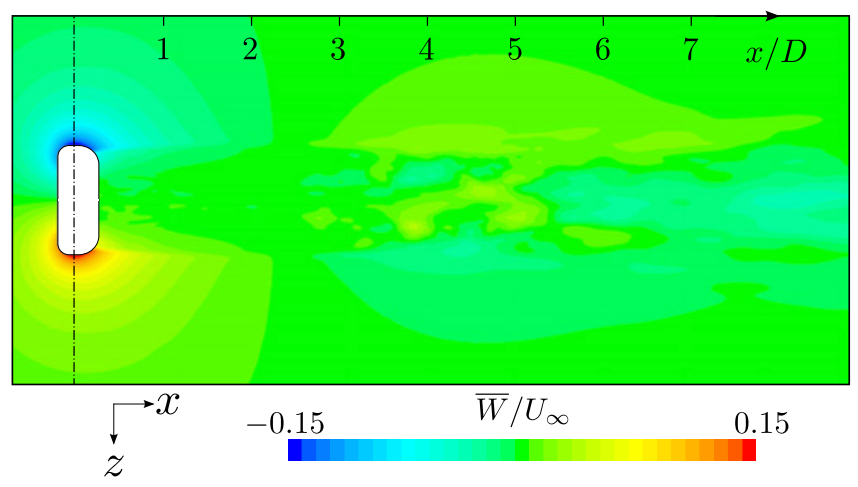

(b) Mean $z$ (radial) velocity component.

Figure 5. Mean velocity components in the wake of the AFT on two planes passing through the turbine's axis of rotation. The white patch corresponds to the area swept by the moving mesh region where the average flow field is not available and the vertical line indicates the location of the turbine's center.

previous studies $[2,48]$. We thus conclude that the AFT wake recovery is not significantly affected by the mean flow field.

\subsection{Cross-flow turbine}

A general view of the CFT wake's vortex dynamics is shown in the visualization of Fig. 6 where the vorticity magnitude is shown through a volume rendering technique. As can be seen in this figure, the streamwise direction is along the $x$ axis, the transverse direction is along the $y$ axis and the spanwise direction is along the $z$ axis. One clearly observes the presence of the tip vortices as well as some spanwise vortex structures. The latter are shed in response to the variation in the blade's bound circulation associated to lift during the blade's revolution. This variation of effective lift is also reflected in the tip-vortex intensity which must vary accordingly. Indeed, Fig. 7 which presents the evolution of the tip vortex circulation during the upstream part of the turbine cycle clearly shows a non-constant intensity.

This circulation has been measured at some instants of the turbine's cycle by computing the vorticity flux through appropriate plane sections encompassing the tip vortex. Care has been taken to assert the independence of the measured 
circulation with respect to the choice and size of the plane sections used. The circulation has only been determined in the upstream half of the cycle because of the difficulty in discriminating between the tip vortices shed in the downstream half and those shed in the upstream half that have convected downstream.

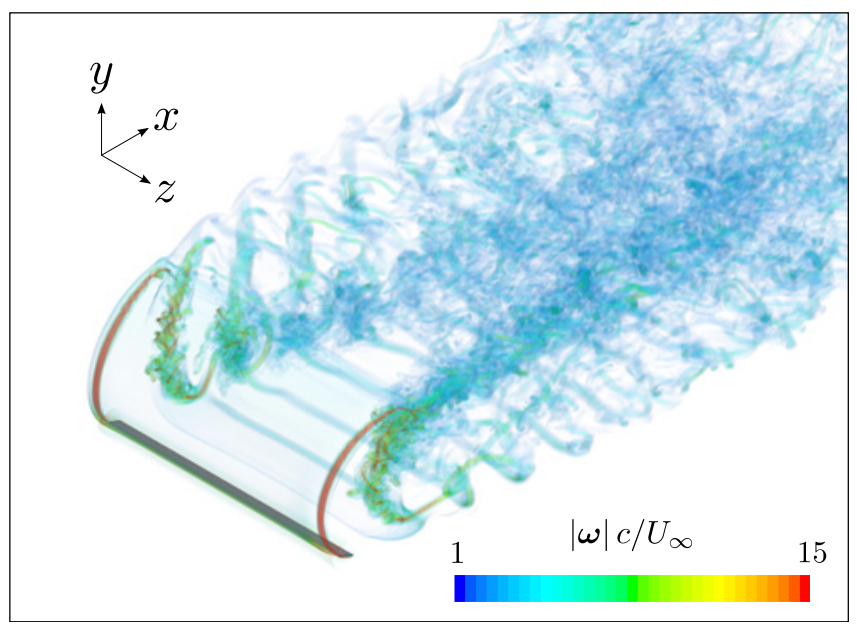

Figure 6. Volume rendering of the vorticity magnitude in the wake of the cross-flow turbine. At the instant shown, the blade is located at $\theta \approx 160^{\circ}$.

It is found that the tip vortices are especially strong when the blade is located near the most upstream location $\left(\theta \approx 90^{\circ}\right)$, where the highest angles of attack are reached [18], as was also observed by Ferreira et al. [7] and by Dixon et al. [51]. The blade's bound circulation is expected to be much smaller and almost constant in the whole downstream-half of the cycle. Indeed, this is supported by the power coefficient cycle of the CFT investigated in this work which was predicted as almost constant with a small negative value in the range $180^{\circ}<\theta<360^{\circ}[22]$.

This is consistant with the fact that the local angle of attack is much smaller there than in the upstream-half of the cycle both because of the significant decrease in the streamwise velocity component and the increase in the transverse velocity component pointing outward (positive $y$ velocity in the $y / D>$ 0 region and negative $y$ velocity in the $y / D<0$ region). All this clearly suggests that the blade's bound circulation value in this fraction of the cycle (downstream-half) should be almost constant and small, as was actually reported by Ferreira et al. [7]. Consequently, the tip vortices should be much weaker in the downstream-half of the cycle and the wake flow field is therefore expected to be mainly affected by the tip vortices generated in the upstream-half of the cycle.

The velocity induced by the strong tip vortices in the middle of the upstream-half cycle results in the spanwise contraction of the wake's central region $\left(y / D \approx 0 ; \theta \approx 90^{\circ}\right)$. This is visible on Fig. 8 which shows the contours of the mean streamwise velocity on various planes perpendicular to the streamwise direction. Combining the effect of the curved paths of the tip vortices and their weaker intensity, as shown

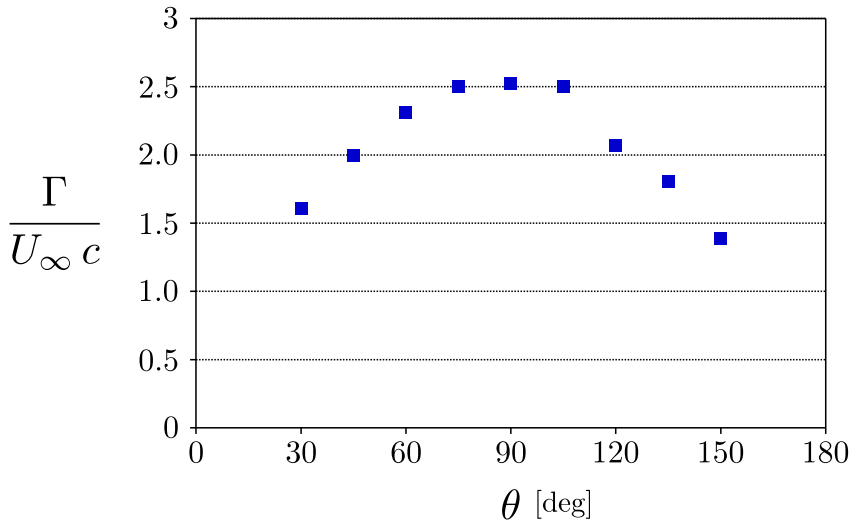

Figure 7. Circulation of the CFT blade's tip vortex at various instants during the upstream-half of the turbine's cycle.

in Fig. 7, at the top and bottom positions (when the blade is located at $\theta \approx 0^{\circ}$ and $\theta \approx 180^{\circ}$ ), the maximum induced spanwise velocity must be observed in the central region. As a result, the wake's spanwise extent contracts in the central part and widens at both transverse edges. As for the transverse extent of the wake, it is found that the wake widens over the whole span. It is worth mentioning that the contractions and the expansions of the wake discussed above in the different regions of the flow field are in agreement with what has already been reported in the literature [7, 10, 52].

One also notices in Fig. 8 that the wake is asymmetric in the $y$ direction with a velocity deficit that is both more pronounced and wider for the upcoming blade region (top of the figures) than for the retreating blade region (bottom of the figures). Battisti et al. [53] and Bachant \& Wosnik [52] both also observed a very similar asymmetry on planes perpendicular to the streamwise direction in the very near wake $(x / D=1.5$ and $x / D=1)$ of three-bladed H-Darrieus turbines having a turbine's aspect ratio value $(b / D)$ around 1.4 and 1 , respectively.

Another important characteristic of the mean streamwise flow field specific to the CFT's wake is the fact that, from approximately $2.25 \mathrm{D}$ downstream of the turbine's center and further downstream, the mean velocity deficit profiles exhibit two local minima separated by a region of higher mean streamwise velocity in the wake center, unlike the AFT's wake. Again, this is due to the spanwise contraction of the wake's central region.

We observed earlier that no significant mean transverse velocity components were present in the wake of the axial-flow turbine. This is not the case anymore for the cross-flow turbine. Indeed, considerable spanwise $(z)$ and transverse $(y)$ mean velocity components are observed in the CFT's wake, as shown in Figs. 9a and 9b, respectively. Indeed, mean transverse and spanwise velocity components respectively reaching up to $25 \%$ and $40 \%$ of the freestream velocity $\left(U_{\infty}\right)$ are observed on the spanwise midplane and the transverse midplane $(z / b=0$ and $y / D=0)$.

This confirms the importance of the mean flow in the spanwise direction on the whole CFT's wake behavior. Con- 
$x / D=1$

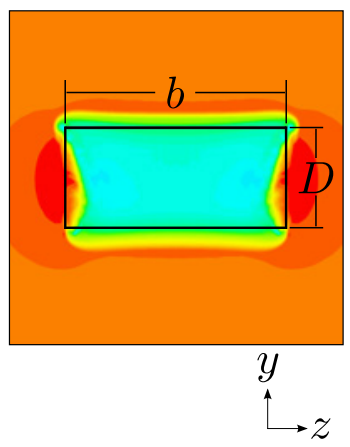

$x / D=3$

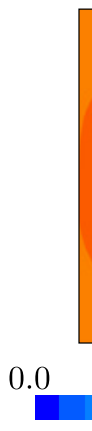

$x / D=5$

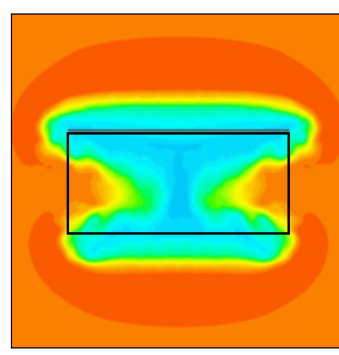

$x / D=7$

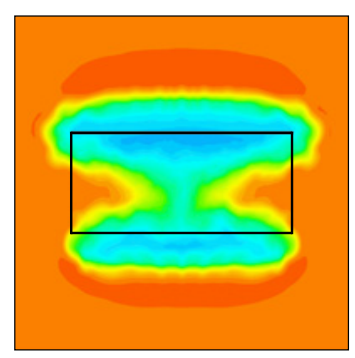

Figure 8. Mean streamwise velocity in the wake of the CFT on various planes perpendicular to the streamwise direction. Note that the black rectangle corresponds to the shape of the turbine's extraction plane and that the upcoming blade region is located at the top of the figure while the retreating blade region is located at the bottom.

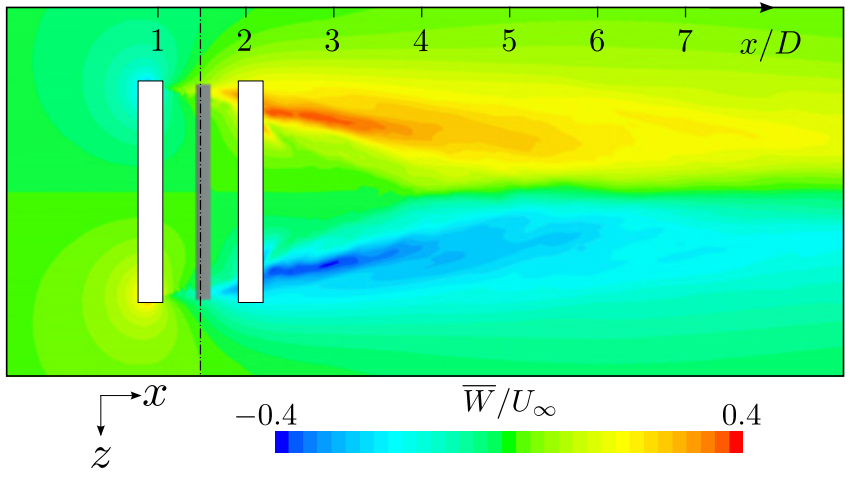

(a) Mean spanwise velocity on the transverse midplane $(y / D=0)$.

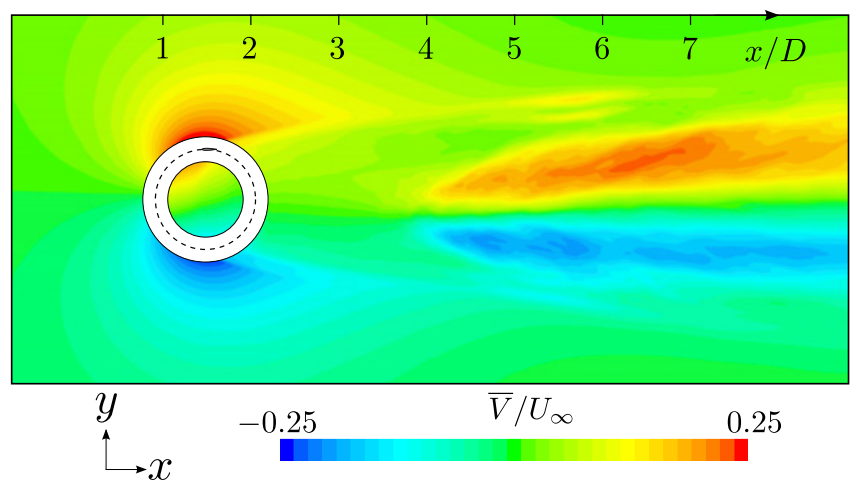

(b) Mean transverse velocity on the spanwise midplane $(z / b=0)$.

Figure 9. Mean velocity components in the wake of the CFT. The white patch corresponds to the area swept by the moving mesh region where the average flow field is not available and the vertical line indicates the location of the turbine's axis of rotation.

sequently, the turbine's aspect ratio $(b / D)$ is expected to be a critical parameter regarding the wake dynamics of this technology. Also, its sensitivity to the free-stream turbulence level is expected to be much weaker than for the axial-flow turbines since it does not depend primarily on the development of a flow instability.

\subsection{Oscillating-foil turbine}

Similarly to the case of the CFT, the angle of attack in the blade's reference frame varies during a cycle of the OFT. Consequently, in addition to tip vortices which are characterized by the fact that their circulation varies throughout the cycle, spanwise vortices are also shed as a result of the variations in the blade's bound circulation. The wake dynamics of the OFT is shown in Fig. 10.

One observes that, in the near wake, the spanwise vortices and the tip vortices form structures similar to vortex loops with their ends tending to wrap around adjacent vortex loops ends (e.g., see the vortex loops labelled as L1 and L2 in Fig. 11). A very similar wake topology has been observed by Dong et al. [13] in the case of a flapping foil in propulsion regime with a blade aspect ratio of 5.09. Each vortex loop is inclined with respect to the streamwise direction resulting in a velocity induced by each vortex loop pointing upstream and outside the wake in the transverse direction, as shown by the dashed arrows in Fig. 11. Further downstream, the spanwise

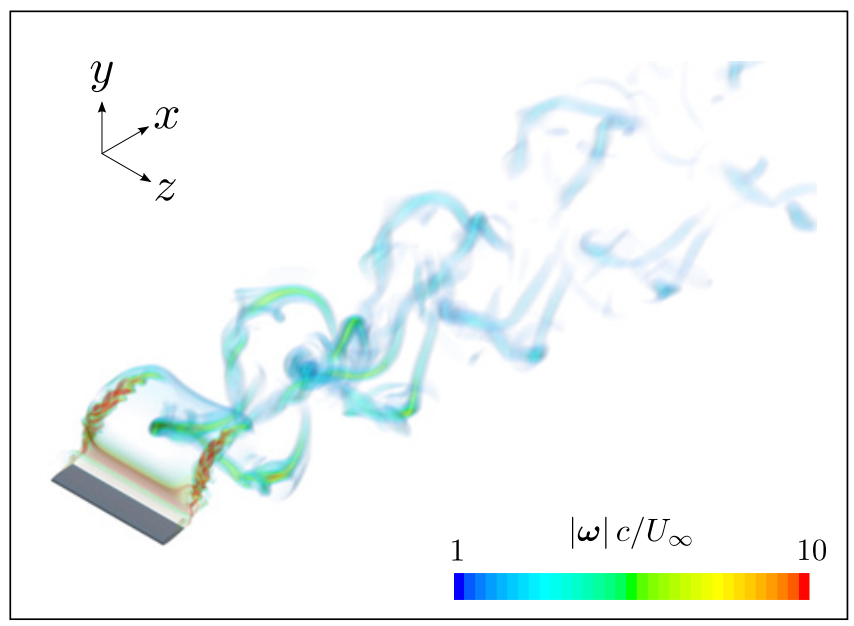

Figure 10. Volume rendering of the vorticity magnitude in the wake of the OFT. At the instant shown, the blade is located at its lowest position reached during a turbine's cycle. 


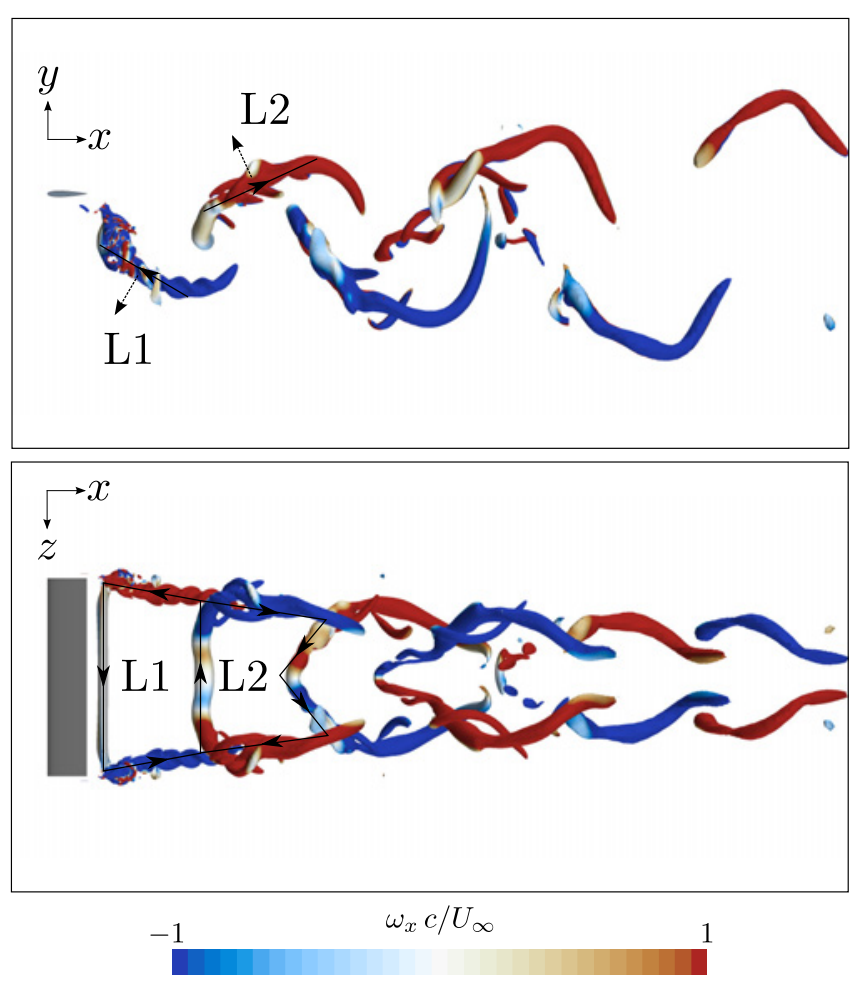

Figure 11. Q-criterion isosurfaces [54] colored by the streamwise vorticity component in the wake of the OFT. The blade is located at its highest position reached during a turbine's cycle. Isosurfaces are not shown in the moving mesh region for clarity.

vortex structures become weaker and only the structures originating from the tip vortices are observed. This suggests that the wake dynamics in this region is dominated by the effects of the tip vortices and is therefore strongly dependent on the blade's aspect ratio, as is also the case for the CFT.

The component of the velocity induced by the vortex loops pointing outside the wake in the transverse direction is responsible for the spreading of the wake in that direction. On the wake centerline, this velocity induction cancels out on average. However, the tip vortices inducing a negative transverse velocity move in the $y / D<0$ direction as they are convected downstream while the tip vortices inducing a positive transverse velocity move in the $y / D>0$ direction. This is clearly visible on Fig. 11 and this results in a significant positive mean transverse velocity component in the $y / D>0$ region of the wake and a negative mean transverse velocity component in the $y / D<0$ region, with a maximum absolute value reaching about $40 \%$ of the freestream velocity, as observed in Fig 12a.

Since the vortex loops follow two oblique paths, the tip vortices on both of these paths induce a mean negative velocity in the $z / b>0$ region of the wake located between these two oblique paths and a mean positive velocity in the $z / b<0$ region. The topology of the resulting mean spanwise velocity field on the traverse midplane $(y / D=0)$ of the OFT is shown in Fig. 12b. It is found that the topology is very similar to that of the CFT (see Fig. 9a) with mean spanwise velocity values reaching approximately $20 \%$ of the freestream velocity.

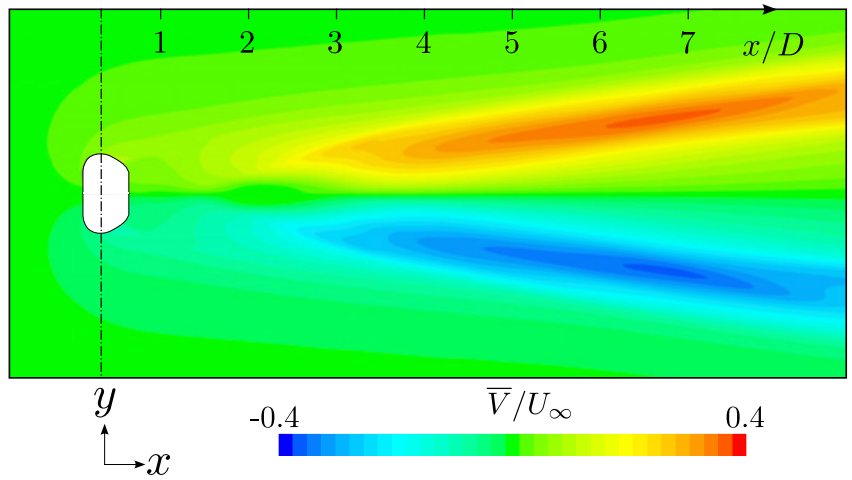

(a) Mean transverse velocity on the spanwise midplane $(z / b=0)$.

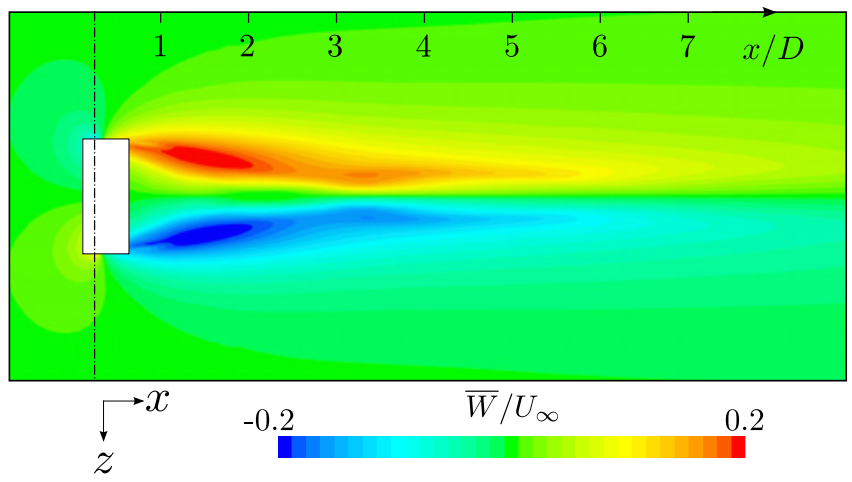

(b) Mean spanwise velocity on the transverse midplane $(y / D=0)$.

Figure 12. Mean velocity components in the wake of the OFT. The white patch corresponds to the area swept by the moving mesh region where the average flow field is not available and the vertical line indicates the location of the turbine's center.

As a result, the OFT's wake central region $(y / D \approx 0)$ contracts in the spanwise direction while it widens in the transverse direction, in agreement with what has been observed by Blondeaux et al. [12] and Dong et al. [13] in their studies of flapping foils as well as by Buchholz \& Smits [14, 15] for a pitching panel, all in propulsion regime. This behavior is also very similar to that of the CFT's wake (see Figs 9a and 9b). It is important to note that the spanwise contraction of the wake due to the velocity induced by the tip vortices results in a decrease in the spanwise vorticity while the latter lead to the stretching of the tip vortices thereby increasing their vorticity, as mentioned by Dong et al. [13]. Indeed, the spanwise vortices located at the upstream end of the tip vortices induce a flow field which tends to stretch the downstream end of the adjacent tip vortices located upstream.

The combined weakening of the spanwise vortex structures along with the intensification of the tip vortices explain why the only significant vortex structures remaining in the far wake are oriented in a direction similar to that of the tip vortices. Note that this behavior is observed despite the fact that the circulation of the spanwise vortex structure is twice that of the vortex structures stemming from the tip vortices. This last 
$x / D=1$

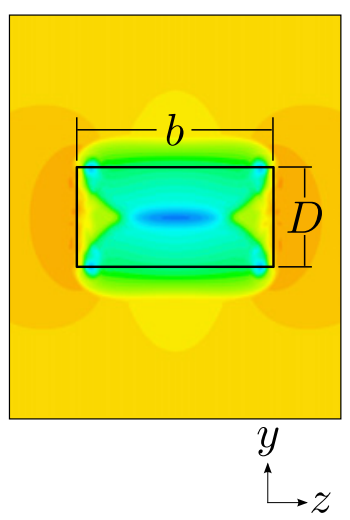

$x / D=3$

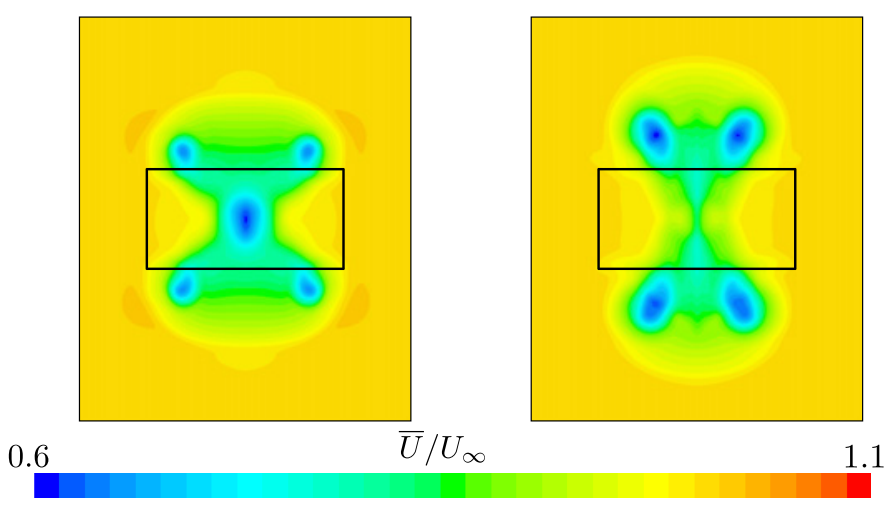

$x / D=7$

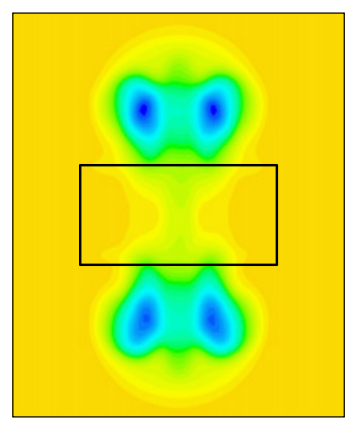

Figure 13. Mean streamwise velocity in the wake of the OFT on various planes perpendicular to the streamwise direction. Note that the black rectangle corresponds to the shape of the turbine's extraction plane.

comment can be understood by observing that each spanwise vortex structures somehow shares two vortex loops or by noting that the blade's bound circulation evolution during a turbine's cycle follows a sinusoidal pattern so that the total circulation variation during half a cycle, which is related to the circulation of the spanwise vortex structures, is equal to twice the amplitude of the circulation's evolution, the latter being related to the circulation of the tip vortices.

Fig. 13 shows the mean streamwise velocity contours on planes perpendicular to the freestream flow located at several positions downstream of the turbine. In the very near wake, the maximum mean velocity deficit is observed in the wake's center. However, beyond approximately 5 turbine's characteristic lengths downstream of the turbine's center, the mean velocity deficit region bifurcates into distinct mean velocity deficit regions which move away from the wake centerline as we travel downstream. Actually, four distinct velocity deficit regions are observed at the corners of the wake. These regions are associated to the negative streamwise velocity induced by the tip vortices resulting from their inclination with respect to the streamwise direction (see Fig. 11).

Note that, since the lift force would be reversed in the propulsion regime compared to the energy extraction regime, the vorticity sign in the vortex loops would also be reversed. As a result, the velocity induced by the tip vortices would be in the opposite direction as the dashed arrow shown in Fig. 11 [13]. The vortex loop labelled L1 would therefore be convected in the $y / D>0$ part of the wake while the vortex loop L2 would be convected in the $y / D<0$ region leading to a pattern similar to the inverted von Kármán vortex street [55]. Because of this difference between these two regimes, Dong et al. [13] noted the presence of two oblique jets in the wake of a flapping foil used for propulsion instead of two velocity deficit regions as observed in the current study.

A similar bifurcation of the wake has also been observed by Blondeaux et al. [12] in the wake of a flapping foil and by Buchholz \& Smits $[14,15]$ in the wake of a pitching panel, both having a finite aspect ratio and used for propulsion at low Reynolds number $\left(\operatorname{Re} \sim 10^{2}-10^{3}\right)$. However, this characteristic of the wake had never been reported before at such a high Reynolds number $\left(\operatorname{Re}_{D}=10^{7}\right)$ and for an oscillating foil in the energy extraction regime. Lastly, Dong et al. [13] also compared the three-dimensional wake of a flapping foil to the two-dimensional wake of the foil operating under the same conditions and found that the bifurcation was only observed in the three-dimensional case. A two-dimensional simulation carried out by the authors showed the same behavior, which can be explained by the fact that tip vortices do not exist in a two-dimensional simulation.

\section{CONCLUSION}

The complete three-dimensional flow field of an axial-flow turbine, a cross-flow turbine and an oscillating-foil turbine have been simulated through the use of the Delayed DetachedEddy Simulation approach. A volume rendering technique illustrating the vorticity magnitude has been used to highlight the dominant vortex structures in the wake of these three different generic technologies in order to develop a better understanding of the main processes affecting their wake recovery.

The main physical mechanisms affecting the wake dynamics of the axial-flow turbine have been found to be notably different from the one affecting the two other types of turbine. Indeed, the axial-flow turbine's wake has been found to be strongly dependent on the triggering of an instability, which is expected to be highly sensitive to the turbulent characteristics of the oncoming flow. The two non-axial turbines' wakes are rather mostly governed by the mean flow field coming from both tips of the blade and directed toward the wake center as a result of the velocity induced by the tip vortices. It is thus reasonable to infer that the wakes of the CFT and the OFT are much less sensitive to the turbulence level of the oncoming flow, but greatly dependent on the geometric characteristics of the turbines. In particular, the turbine's aspect ratio appears as a critical characteristic, i.e, the ratio of the blade span over the turbine's diameter for the case of the CFT, and the ratio of 
the blade span over the overall extent of the blade motion for the case of the OFT.

Future works will be devoted to the assessment of the relative importance of each physical mechanism affecting the wake recovery of the three turbine concepts.

\section{ACKNOWLEDGMENTS}

Financial support from the Natural Sciences and Engineering Research Council of Canada (NSERC) and the Fonds de recherche du Québec - Nature et technologies (FRQ-NT) is gratefully acknowledged by the authors. Computations in this work were run on the supercomputers Guillimin from McGill University and Colosse from Laval University, both managed by Calcul Québec and Compute Canada.

\section{REFERENCES}

[1] Michael Sherry, Andras Nemes, David Lo Jacono, Hugh M. Blackburn, and John Sheridan. The interaction of helical tip and root vortices in a wind turbine wake. Physics of Fluids, 25(11):117102 (16 pp.), 2013.

[2] L. P. Chamorro, Daniel R. Troolin, Seung-Jae Lee, R. E. A. Arndt, and Fotis Sotiropoulos. Three-dimensional flow visualization in the wake of a miniature axial-flow hydrokinetic turbine. Experiments in Fluids, 54(2):(12 pp.), 2013.

[3] L. E. M. Lignarolo, D. Ragni, C. Krishnaswami, Q. Chen, C. J. Simão Ferreira, and G. J. W. van Bussel. Experimental analysis of the wake of a horizontal-axis wind-turbine model. Renewable Energy, 70:31 - 46, 2014.

[4] C. J. Simão Ferreira, Gerard J. W. Van Bussel, and Gijs A. M. Van Kuik. Wind Tunnel Hotwire Measurements, Flow Visualization and Thrust Measurement of a VAWT in Skew. Journal of Solar Energy Engineering, Transactions of the ASME, 128(4):487 - 497, 2006.

[5] C. J. Simão Ferreira, Gerard Van Bussel, Fulvio Scarano, and Gijs Van Kuik. 2D PIV visualization of dynamic stall on a vertical axis wind turbine. In Collection of Technical Papers - 45th AIAA Aerospace Sciences Meeting, volume 23, pages 16175 - 16190, Reno, NV, United states, 2007.

[6] C. J. Simão Ferreira, G. Kuik, G. Bussel, and F. Scarano. Visualization by PIV of dynamic stall on a vertical axis wind turbine. Experiments in Fluids, 46(1):97 - 108, 2009.

[7] C. J. Simão Ferreira, Claudia Hofemann, Kristian Dixon, Gijs Van Kuik, and Gerard Van Bussel. 3D wake dynamics of the VAWT: Experimental and numerical investigation. In 48th AIAA Aerospace Sciences Meeting Including the New Horizons Forum and Aerospace Exposition, Orlando, FL, United states, 2010.

[8] C. Hofemann, C. J. Simão Ferreira, G. J. Van Bussel, G. A. M. Van Kuik, F. Scarano, and K. R. Dixon. 3D Stereo PIV Study of Tip Vortex Evolution on a VAWT. In
The proceedings of the European Wind Energy Conference Exhibition EWEC, Brussels, Belgium, 2008.

${ }^{\text {[9] }}$ F. Scheurich, T. M. Fletcher, and R. E. Brown. Simulating the aerodynamic performance and wake dynamics of a vertical-axis wind turbine. Wind Energy, 14(2):159 - 77, 2011.

[10] G. Tescione, D. Ragni, C. He, C. J. Simão Ferreira, and G. J. W. van Bussel. Near wake flow analysis of a vertical axis wind turbine by stereoscopic particle image velocimetry. Renewable Energy, 70:47 - 61, 2014.

[11] Jian Deng, C. P. Caulfield, and Xueming Shao. Effect of aspect ratio on the energy extraction efficiency of three-dimensional flapping foils. Physics of Fluids, 26(4):043102 (24 pp.), 2014.

${ }^{\text {[12] }}$ P. Blondeaux, F. Fornarelli, L. Guglielmini, M. S. Triantafyllou, and R. Verzicco. Numerical experiments on flapping foils mimicking fish-like locomotion. Physics of Fluids, 17(11):113601 (12 pp.), 2005.

[13] H. Dong, R. Mittal, and F. M. Najjar. Wake topology and hydrodynamic performance of low-aspect-ratio flapping foils. Journal of Fluid Mechanics, 566:309 - 343, 2006.

[14] J. H. J. Buchholz and A. J. Smits. On the evolution of the wake structure produced by a low-aspect-ratio pitching panel. Journal of Fluid Mechanics, 546:433 - 443, 2006.

[15] J. H. J. Buchholz and A. J. Smits. The wake structure and thrust performance of a rigid low-aspect-ratio pitching panel. Journal of Fluid Mechanics, 603:331 - 365, 2008.

[16] Tony Burton. Wind Energy Handbook. Wiley, Chichester, New York, $2^{\text {nd }}$ edition, 2011.

[17] J. N. Sørensen. Aerodynamic Aspects of Wind Energy Conversion. Annual Review of Fluid Mechanics, 43:427 448, 2011.

[18] I. Paraschivoiu. Wind Turbine Design: With Emphasis on Darrieus Concept. Polytechnic International Press, 2002.

[19] T. Kinsey and G. Dumas. Optimal operating parameters for an oscillating foil turbine at reynolds number 500,000. AIAA Journal, 52(9):1885 - 1895, 2014.

[20] V. Klaptocz, C. Crawford, M. Shives, I. Franchini, and E. Mete Sireli. Impact of channel blockage, free surface proximity and foundations on the performance of Tidal/River Energy Converters. Technical report, Marine Energy Standards TC114, 2014.

[21] Marine Renewables Canada. Marine Renewables Canada (OREG). http://www.marinerenewables.ca/ (accessed on 2015/01/08)

[22] R. Gosselin, G. Dumas, and M. Boudreau. Parametric Study of H-Darrieus vertical-axis turbines using uRANS simulations. In 21st Annual Conference of the CFD Society of Canada, Sherbrooke, Quebec, Canada, 2013.

[23] Thomas Kinsey and Guy Dumas. Three-Dimensional Effects on an Oscillating-Foil Hydrokinetic Turbine. Journal of Fluids Engineering, 134(7):071105 (11 pp.), 2012. 
[24] Peter Bachant and Martin Wosnik. Reynolds Number Dependence of Cross-Flow Turbine Performance and Near-Wake Characteristics. In Proceedings of the $2^{\text {nd }}$ Marine Energy Technology Symposium, page (9 pp.), 2014.

[25] Leonardo P. Chamorro, R. E. A. Arndt, and F. Sotiropoulos. Reynolds number dependence of turbulence statistics in the wake of wind turbines. Wind Energy, 15(5):733 $742,2012$.

[26] Pierre-Elouan Rethore. Wind Turbine Wake in Atmospheric Turbulence. $\mathrm{PhD}$ thesis, Aalborg University, Denmark, 2009.

[27] P. R. Spalart. Detached-Eddy Simulation. Annual Review of Fluid Mechanics, 41:181-202, 2009.

[28] P. R. Spalart, S. Deck, M. L. Shur, K. D. Squires, M. Kh. Strelets, and A. Travin. A New Version of Detachededdy Simulation, Resistant to Ambiguous Grid Densities. Theoretical and Computational Fluid Dynamics, 20(3):181-195, 2006.

[29] P. R. Spalart and S. R. Allmaras. A one-equation turbulence model for aerodynamic flows. La Recherche Aérospatiale, 1:5 - 21, 1994.

[30] M. Shur, P. R. Spalart, M. Strelets, and A. Travin. Detached-eddy simulation of an airfoil at high angle of attack. In Engineering turbulence modelling and experiments 4, Proceedings of the 4th International Symposium on Engineering Turbulence Modelling and Measurements, pages 669-678, Ajaccio, Corsica, France, 1999.

[31] P. R. Spalart, W-H. Jou, M. Strelets, and S. R. Allmaras. Comments on the Feasibility of LES for Wings, and on a Hybrid RANS/LES Approach. In Advances in DNS/LES, Proceedings of the First AFOSR International Conference on DNS/LES, pages 137 - 147, Ruston, Louisiana, USA, 1997.

[32] CD-Adapco ${ }^{\text {TM }}$ STAR-CCM+® V9. User's Guide. http: //www. cd-adapco.com/products/star-ccm, 2014.

[33] A. Travin, M. Shur, M. Strelets, and P.R. Spalart. Physical and Numerical Upgrades in the Detached-Eddy Simulation of Complex Turbulent Flows. In Advances in LES of Complex Flows, volume 65, pages 239 - 254, 2002.

[34] T. Kinsey and G. Dumas. Impact of channel blockage on the performance of axial and cross-flow hydrokinetic turbines. 2015. (To appear).

[35] Philippe R. Spalart and Christopher L. Rumsey. Effective inflow conditions for turbulence models in aerodynamic calculations. AIAA Journal, 45(10):2544 - 2553, 2007.

[36] P. R. Spalart. Young-Person's Guide to Detached-eddy Simulation Grids. Technical report, NASA contractor report, 2001.

[37] Charles Mockett, Rodolphe Perrin, Thorsten Reimann, Marianna Braza, and Frank Thiele. Analysis of DetachedEddy Simulation for the Flow Around a Circular Cylinder with Reference to PIV Data. Flow, Turbulence and Combustion, 85(2):167 - 180, 2010.

[38] T. Kinsey and G. Dumas. Computational fluid dynamics analysis of a hydrokinetic turbine based on oscillating hydrofoils. Journal of Fluids Engineering, 134(2):021104 (16 pp.), 2012.

[39] Jang-Oh Mo, Amanullah Choudhry, Maziar Arjomandi, and Young-Ho Lee. Large eddy simulation of the wind turbine wake characteristics in the numerical wind tunnel model. Journal of Wind Engineering and Industrial Aerodynamics, 112:11 - 24, 2013.

[40] Philippe Chatelain, Stéphane Backaert, Grégoire Winckelmans, and Stefan Kern. Large Eddy Simulation of Wind Turbine Wakes. Flow, Turbulence and Combustion, 91(3):587 - 605, 2013.

[41] Seokkoo Kang, Iman Borazjani, Jonathan A. Colby, and Fotis Sotiropoulos. Numerical simulation of 3D flow past a real-life marine hydrokinetic turbine. Advances in Water Resources, 39:33 - 43, 2012.

[42] S. C. Tedds, I. Owen, and R. J. Poole. Near-wake characteristics of a model horizontal axis tidal stream turbine. Renewable Energy, 63:222 - 235, 2014.

[43] Majid Bastankhah and Fernando Porté-Agel. A new analytical model for wind-turbine wakes. Renewable Energy, 70:116 - 123, 2014.

${ }^{[44]}$ L. P. Chamorro and F. Porté-Agel. A Wind-Tunnel Investigation of Wind-Turbine Wakes: Boundary-Layer Turbulence Effects. Boundary-Layer Meteorology, 132(1):129 $-149,2009$.

[45] Chia-Ren Chu and Pei-Hung Chiang. Turbulence effects on the wake flow and power production of a horizontalaxis wind turbine. Journal of Wind Engineering and Industrial Aerodynamics, 124:82 - 89, 2014.

[46] D. Medici and P. H. Alfredsson. Measurements on a wind turbine wake: 3D effects and bluff body vortex shedding. Wind Energy, 9(3):219 - 236, 2006.

[47] B. Sanderse, S. P. van der Pijl, and B. Koren. Review of computational fluid dynamics for wind turbine wake aerodynamics. Wind Energy, 14(7):799 - 819, 2011.

[48] Wei Zhang, C. D. Markfort, and F. Porté-Agel. Near-wake flow structure downwind of a wind turbine in a turbulent boundary layer. Experiments in Fluids, 52(5):1219 - 1235, 2012.

[49] M. Felli, R. Camussi, and F. Di Felice. Mechanisms of evolution of the propeller wake in the transition and far fields. Journal of Fluid Mechanics, 682:5 - 53, 2011.

[50] Paul Mycek, Benoît Gaurier, Grégory Germain, Grégory Pinon, and Elie Rivoalen. Experimental study of the turbulence intensity effects on marine current turbines behaviour. Part II: Two interacting turbines. Renewable Energy, 68:876 - 892, 2014. 
[51] K.R. Dixon, C.J. Simão Ferreira, Claudia Hofemann, Gerard Van Bussel, and Gijs Van Kuik. A 3d unsteady panel method for vertical axis wind turbines. In The proceedings of the European Wind Energy Conference Exhibition EWEC, pages 2981 - 2990, Brussels, Belgium, 2008.

[52] Peter Bachant and Martin Wosnik. Characterising the near-wake of a cross-flow turbine. Journal of Turbulence, 16(4):392 - 410, 2015.

[53] L. Battisti, L. Zanne, S. Dell'Anna, V. Dossena, G. Persico, and B. Paradiso. Aerodynamic Measurements on a Vertical Axis Wind Turbine in a Large Scale Wind Tunnel. Journal of Energy Resources Technology, 133(3):031201 (9 pp.), 2011.

[54] J. C. R. Hunt, A. A. Wray, and P. Moin. Eddies, Streams, and Convergence Zones in Turbulent Flows. In Center for Turbulence Research, Proceedings of the 1988 Summer Program, pages 193 - 208, 1988.

[55] T. Schnipper, A. Andersen, and T. Bohr. Vortex wakes of a flapping foil. Journal of Fluid Mechanics, 633:411 423, 2009. 